\title{
BMJ Open How many people will need palliative care in Scotland by 2040? A mixed- method study of projected palliative care need and recommendations for service delivery
}

\author{
Anne M Finucane (D) ,1,2 Anna E Bone, ${ }^{3}$ Simon Etkind, ${ }^{3}$ David Carr, ${ }^{4}$ \\ Richard Meade, ${ }^{5}$ Rosalia Munoz-Arroyo, ${ }^{4}$ Sébastien Moine, ${ }^{2,6}$ \\ Aghimien lyayi-Igbinovia, ${ }^{4}$ Catherine J Evans, ${ }^{3}$ Irene J Higginson, ${ }^{3}$ \\ Scott A Murray (i) ${ }^{2}$
}

To cite: Finucane AM, Bone AE, Etkind $\mathrm{S}$, et al. How many people will need palliative care in Scotland by 2040? A mixed-method study of projected palliative care need and recommendations for service delivery. BMJ Open 2021;11:e041317. doi:10.1136/ bmjopen-2020-041317

- Prepublication history and additional material for this paper is available online. To view these files, please visit the journal online (http://dx.doi.org/10. 1136/bmjopen-2020-041317).

Received 04 June 2020 Revised 08 December 2020 Accepted 05 January 2021

\section{A Check for updates}

(c) Author(s) (or their employer(s)) 2021. Re-use permitted under CC BY-NC. No commercial re-use. See rights and permissions. Published by BMJ.

For numbered affiliations see end of article.

\section{Correspondence to}

Dr Anne M Finucane; anne.finucane@mariecurie. org.uk

\section{ABSTRACT}

Objective To estimate future palliative care need and complexity of need in Scotland, and to identify priorities for future service delivery.

Design We estimated the prevalence of palliative care need by analysing the proportion of deaths from defined chronic progressive illnesses. We described linear projections up to 2040 using national death registry data and official mortality forecasts. An expert consultation and subsequent online consensus survey generated recommendations on meeting future palliative care need. Setting Scotland, population of 5.4 million.

Participants All decedents in Scotland over 11 years (2007 to 2017). The consultation had 34 participants; 24 completed the consensus survey.

Primary and secondary outcomes Estimates of past and future palliative care need in Scotland from 2007 up to 2040. Multimorbidity was operationalised as two or more registered causes of death from different disease groups (cancer, organ failure, dementia, other). Consultation and survey data were analysed descriptively.

Results We project that by 2040 , the number of people requiring palliative care will increase by at least $14 \%$; and by $20 \%$ if we factor in multimorbidity. The number of people dying from multiple diseases associated with different disease groups is projected to increase from $27 \%$ of all deaths in 2017 to $43 \%$ by 2040 . To address increased need and complexity, experts prioritised sustained investment in a national digital platform, roll-out of integrated electronic health and social care records; and approaches that remain person-centred.

Conclusions By 2040 more people in Scotland are projected to die with palliative care needs, and the complexity of need will increase markedly. Service delivery models must adapt to serve growing demand and complexity associated with dying from multiple diseases from different disease groups. We need sustained investment in secure, accessible, integrated and personcentred health and social care digital systems, to improve care coordination and optimise palliative care for people across care settings.

\section{Strengths and limitations of this study}

- First study to project estimates of future palliative care need for Scotland.

- Used a range of estimation methods, accounting for both underlying and contributory causes of death, to produce robust projections.

- The expert consultation and consensus survey ensured that recommendations were grounded in practice.

- We drew on death registry data that relies on accurate completion of death certificates.

- Our definition of multimorbidity was relatively restrictive - decedents were required to have two major illnesses from different disease groups, both of which would individually confer palliative care need.

\section{BACKGROUND}

Worldwide, deaths are expected to rise from 55 million in 2016 to 75 million by $2040 .{ }^{1}$ Non-communicable diseases accounted for $72 \%$ of global deaths in 2016, and are forecast to account for $81 \%$ of deaths by $2040 .{ }^{1}$ Globally, the proportion of people aged 80 or over increased by $76.5 \%$ between 2000 and $2015 .^{2}$ The growing number of older people and increased prevalence of chronic conditions indicate that more people would benefit from a palliative approach to care over the next two decades.

Demographic changes and shifts in disease patterns at a national level will impact population palliative care need. An analysis based on data from 2006 to 2014 in England and Wales, projected that up to $87.6 \%$ of people who die could benefit from palliative care by $2040 .{ }^{3} \mathrm{In}$ Ireland, a similar analysis based on data from 2007 to 2015, projected that palliative care 
need will increase, and will be indicated for $83 \%$ of those who die by $2046 .{ }^{4}$ However, existing projection studies use data based on 'main underlying cause of death' only, excluding people who may have had a contributory cause of death (but not a main underlying cause) associated with palliative care need. Models that only use "main underlying cause of death' may therefore underestimate the true extent of population palliative care need; models that incorporate both main underlying and contributory causes of death are required.

Increased longevity in developed and developing countries comes with greater multimorbidity. Multimorbidity describes the co-existence of two or more chronic conditions which may be a physical non-communicable disease, a mental health condition or infectious disease of long duration. ${ }^{56}$ Data from Scotland has shown that $65 \%$ of people aged 65 to 84 years have two or more long-term disorders; rising to $81.5 \%$ for people aged 85 and over. ${ }^{7}$ Only $5.3 \%$ of people with dementia have no other longterm disorder. ${ }^{8}$ Despite the recognition that multimorbidity is the norm in high-income countries, ${ }^{5}$ there has been little consideration of the implications for palliative care. To inform future palliative care service design, there is a need to better understand multimorbidity due to the presence of advanced progressive diseases. As a starting point, we need estimates of the number of people dying from multiple diseases associated with palliative care need now and in the future.

The aim of this study was to estimate, and project future palliative care need and complexity of need in Scotland up to 2040. Extending previous work, we sought to estimate and project the number of people dying from more than one disease associated with palliative care need. Furthermore, we planned an expert consultation and consensus survey to generate recommendations for future palliative care service delivery. This novel analysis sought to provide a more comprehensive picture of future palliative care need, and complexity of need, than previously described.

\section{METHODS \\ Design}

Mixed methods consisting of: (1) simple linear modelling of routinely available national death registry data and mortality projections for Scotland and (2) expert consultation and online consensus survey to generate recommendations for service design and delivery based on our projections.

\section{Setting}

Scotland is a high-income Western European country with a population of 5.4 million; 57870 people died in 2017. ${ }^{9}$ Mortality forecasts project that annual deaths will increase by $13.6 \%$ to 65756 by $2040 .^{10}$

\section{Data sources}

Deaths by age, gender and cause from 2007 to 2017

We obtained aggregate level data on deaths by age, gender and cause for an 11-year period (2007 to 2017 inclusive) from Public Health Scotland (previously known as Information Services Division Scotland). The aggregate data were derived from the National Records of Scotland individuallevel deaths database held by Public Health Scotland.

Mortality projections for Scotland are available from the Office for National Statistics and National Records of Scotland. ${ }^{10}{ }^{11}$ The mortality projections used in this analysis were based on the 2016 principal population projections for Scotland up to 2040. We used projections up to 2040 to allow comparison with studies in other countries.

\section{Generating estimates of population palliative care need}

Palliative care need at a population level has been estimated using a variety of methods. Gómez-Batiste et al estimated that $75 \%$ of all those who die have palliative care needs. $^{12}$

Other approaches use disease-specific mortality data and identify diseases using International Classification of Disease $-10^{\text {th }}$ Revision (ICD-10) codes associated with palliative care need to generate estimates. ${ }^{13}{ }^{14}$ Murtagh et al estimated that in high-income countries, $63 \%$ to $97 \%$ of people who die might benefit from palliative care depending on the estimation method used. ${ }^{14}$ Based on this previous work, we estimated and projected palliative care need in three ways as described below.

\section{Estimation method 1: $75 \%$ of all deaths}

Method 1 is based on that used by Gómez-Batiste et al. ${ }^{12}$ It assumes that $75 \%$ of people in high-income countries die from chronic progressive diseases with evolving and increasing healthcare needs. Consequently, $75 \%$ of people at the end of life may benefit from a palliative care approach, irrespective of whether that is delivered by palliative care specialists or other health professionals. We calculated palliative care need as $75 \%$ of all deaths in 2017, and projected this proportion up to 2040.

Estimation method 2: palliative care need based on ICD-10 codes associated with main underlying cause of death

Method 2 takes a diagnosis-based approach, applying ICD-10 diagnostic codes previously used to estimate population palliative care need (online supplemental file 1). ${ }^{314}$ To project future palliative care need, we modelled two scenarios (2A and 2B). For method 2A, we calculated the number and proportion of age and gender-specific deaths in 2017 where an ICD-10 code associated with palliative care was recorded as the main underlying cause of death, then applied this proportion to mortality forecasts for each year up to 2040, with no adjustments. For method 2B we calculated the mean annual change in the proportion of deaths requiring palliative care between 2007 and 2017. We assumed the mean annual change would continue in a linear fashion and applied the resulting proportion to mortality forecasts up to 2040 . We 
chose method 2B for subanalyses projecting future needs by age and disease group as it enabled comparison with data reported in other published studies. ${ }^{34}$

\section{Estimation method 3: palliative care need based on ICD-10 codes} associated with main underlying and all contributory cause(s) of death

Method 3 adopts a diagnostic-based approach, using the same ICD-10 diagnostic codes outlined in method 2 to estimate the number of people dying from diseases associated with palliative care need. However, for method 3, our estimate of palliative care need was the number of people for whom a palliative care relevant disease was recorded as either the main underlying cause of death, or a contributory cause. Thus, method 3 should yield the most accurate and comprehensive estimate of deaths associated with palliative care need in a calendar year. To project future need using this method, we calculated the number and proportion of age and gender-specific deaths from 2007 to 2017 where an ICD-10 code associated with palliative care was recorded, as either the main underlying or contributory cause of death. As for method 2B, we assumed the mean annual change in the proportion of deaths associated with palliative care need over the baseline period would continue to occur in a linear fashion, and applied the resulting mean annual change to mortality forecasts up to 2040.

\section{Defining, estimating and projecting multimorbidity associated with advanced progressive diseases}

We wanted to estimate and project the number of people dying from multimorbidity associated with palliative care need. Given that some diseases are closely related and may represent similar disease processes (e.g. related cancers), we chose to define multimorbidity as people dying from two or more chronic diseases associated with palliative care need from more than one of four disease groups-cancer, organ failure, dementia or other (includes neurological conditions and stroke). This definition indicates care complexity, given the distinct illness trajectories, needs and configuration of services associated with diseases from each group, which when experienced together greatly increase symptom burden and need for care coordination. To generate this estimate, we calculated the mean annual change in the proportion of deaths due to two or more diseases from different disease groups over the 11-year period. We assumed the mean annual change would continue to occur in a linear fashion and applied the resulting mean annual change in the proportion of deaths associated with diseases from different disease groups to mortality forecasts up to 2040.

\section{Expert consultation and consensus survey}

We used an abbreviated MORECare Transparent Expert Consultation process, consisting of a modified nominal group technique, to generate recommendations in response to projected palliative care need. ${ }^{15}$ This was followed by a consensus survey to rank and ascertain consensus on each recommendation for the future delivery of palliative care. A purposive sample of approximately 50 stakeholders from palliative care, primary care and social care along with commissioners, service providers, government representatives, researchers, patient/carer groups and charities were invited to take part. Potential participants were identified by co-authors; some were known to the co-authors and had participated in previous consultations. A half-day consultation took place on 3 September 2019. Projections of future palliative care need, based on the quantitative data analysis, were presented. Participants were assigned to one of four groups designed to maximise group heterogeneity in terms of background, role and experience. Each group discussed the palliative care need projections, critical issues emerging from the data and then generated recommendations in response. Individual participants completed a recommendations form in response to the question 'What actions need to be prioritised to meet future palliative care need by 2040 ', and each group identified the group priorities on a white board during the discussion. Individual and group level priorities were collected. These were subsequently recorded in Microsoft Excel, synthesised and used to develop specific statements for the follow-up consensus survey. One month later, participants received the follow-up survey by email, stating the recommendations generated at the event. Participants were asked to rate the extent to which they agreed with each recommendation from 1 to 9 (strongly disagree to strongly agree), using free-text spaces to add comments explaining their judgements. One completion reminder was sent after 2 weeks.

Survey data was analysed in Excel using descriptive statistics. Informed by guidance on consensus methods, ${ }^{16}$ we categorised each recommendation as follows, to ascertain level of agreement for each statement: scores of 1 to 3 (recommendation not indicated), 4 to 6 (equivocal) and 7 to 9 (recommendation indicated). We then examined the dispersion of scores for each statement to determine degree of consensus. Where the Interquartile Range (IQR) fell within each 3-point region outlined, we judged there to be 'close agreement' for the recommendation; but where ratings fell across each of these regions, we judged that there was 'broad agreement'.

\section{Patient and public involvement}

A member of the Marie Curie Voices group (patient and carer representative group) participated in the expert consultation and survey and shared his perspectives on the meaning of the findings for patient and family care at the event. Three patient and public involvement representatives from the Marie Curie Voices group, and one volunteer, commented on the draft manuscript and survey findings. These comments were used to inform study implications.

\section{Reporting guidelines}

We used the Strengthening the Reporting of Observational Studies in Epidemiology (STROBE) guidelines to inform reporting (see online supplemental file 1). 


\section{RESULTS}

Estimated palliative care need in Scotland 2007 to 2017

During this 11-year period, registered deaths in Scotland rose by $3.4 \%$ (55 984 to 57870 ). ${ }^{9}$ Using various methods to estimate population level palliative care need, we estimated that the number of people who died with palliative care needs in Scotland in 2017 ranged from 43403 (method 1) to 52148 (method 3), an increase from 2007 irrespective of method used (table 1). See online supplemental file 2 for data by year.

\section{Projections of population level palliative care need in Scotland from 2018 to 2040}

Deaths in Scotland are projected to reach 65756 by 2040, a $13.6 \%$ increase from $2017 .{ }^{10}$ Irrespective of the projection method used, the number of people dying of disease(s) associated with palliative care need is projected to increase. By 2040, we estimate that between $74 \%$ and $95 \%$ of those who die might benefit from a palliative care approach (table 1). See online supplemental file 2 for data by year.

\section{Projections of palliative care need by age group up to 2040}

Based on projection method 2B, the number of people who die aged 0 to 74 years with palliative care needs is projected to fall, while those aged 75 and over will increase (figure 1). The greatest increase will occur for those aged 85 and above. People aged 85 or older will account for just under half $(47 \%)$ of those dying with palliative care needs by 2040 (23 491 of 50084 projected deaths associated with palliative care need).

\section{Projections of underlying cause of death by disease group up} to $\mathbf{2 0 4 0}$

Based on projection method 2B, deaths with cancer as the main underlying cause of death are projected to rise from 16203 in 2017 to 19535 in 2040 (21\% increase). Deaths where dementia is the main underlying cause of death are projected to rise from 6776 in 2017 to 19284 (185\% increase); while deaths from organ failure are projected to continue to fall (from 14928 in 2017 to 9957 by 2040) as are deaths from other conditions requiring palliative care (4909 in 2017 to 1668 in 2040) (figure 2).

\section{Proportion of people dying with multimorbidity associated with advanced progressive diseases across different disease groups}

In 2017, overall, 15725 people died with multimorbidity, that is, at least two diseases associated with palliative care need from different disease groups (27.2\% of all deaths). The number of people projected to die with multimorbidity is projected to increase by $82 \%$ to 28629 by 2040 , accounting for $43.5 \%$ of all deaths or $45.6 \%$ of all palliative care deaths using projection method 3 (figure 3 ). The proportion of those who die with multimorbidity is projected to increase by $60 \%$ ( $27.2 \%$ to $43.5 \%)$. The increases will mainly occur for those in the older age groups (figure 4). If current trends continue, by 2040, we project that $52 \%$ of those aged 85 to 99 will die from two

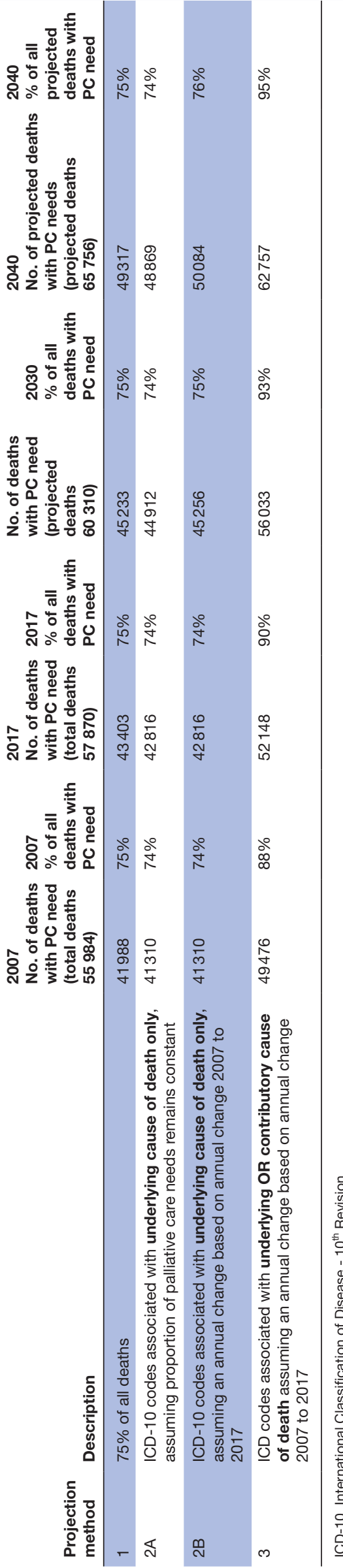




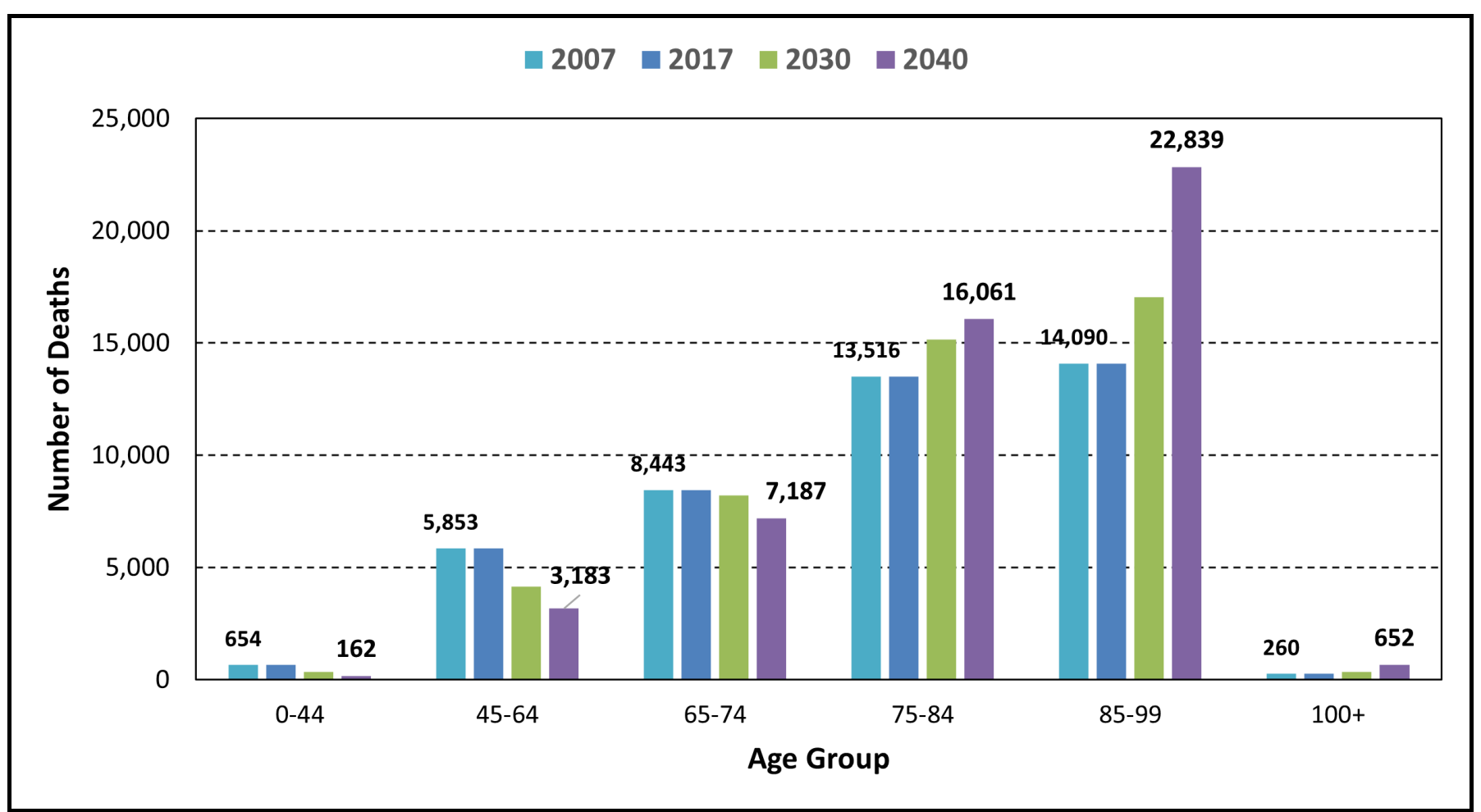

Figure 1 Projected estimates of the number of people dying in Scotland with palliative care needs by age up to 2040 using method 2B. Note: Data for 2007 and 2017 is actual deaths; data for 2030 and 2040 is projected deaths based on method 2B.

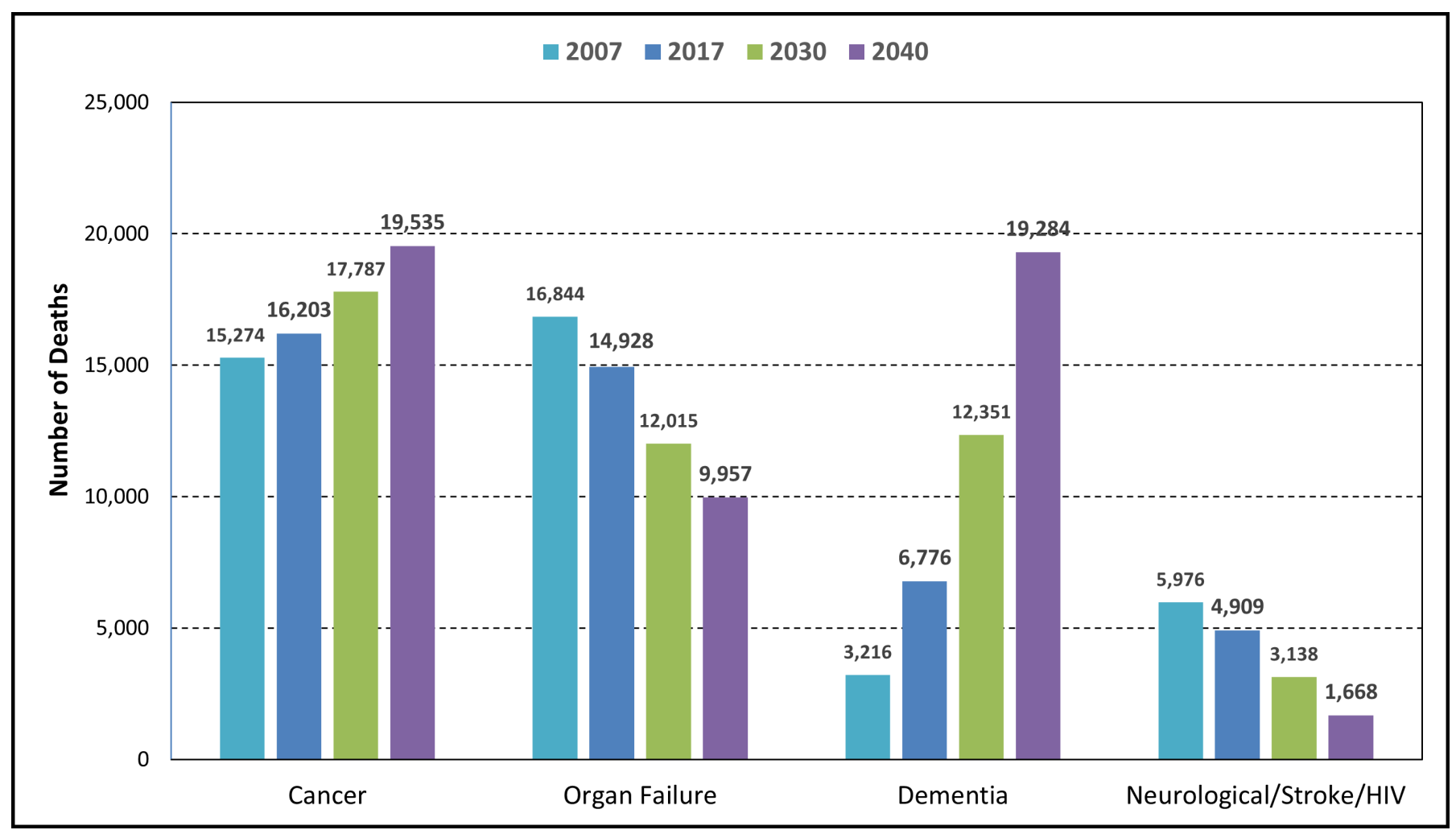

Figure 2 Projected main underlying cause of death associated with palliative care need by disease group up to 2040 using method 2B. Note: Data for 2007 and 2017 is actual deaths; data for 2030 and 2040 is projected deaths based on method 2B. 


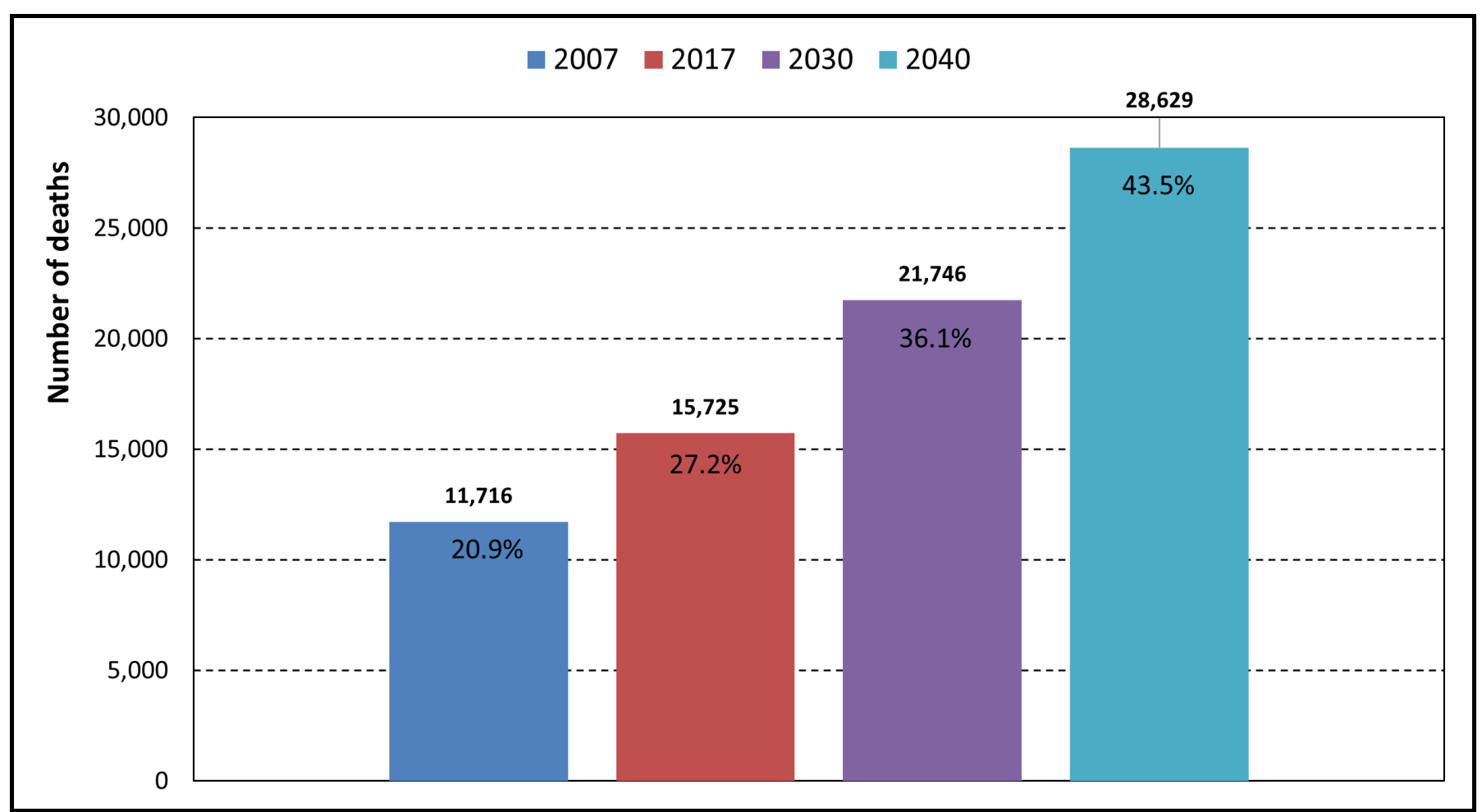

Figure 3 Projected number and percentage of people in Scotland dying from multimorbidity associated with palliative care need, 2017 to 2040. Note: Data for 2007 and 2017 is actual deaths; data for 2030 and 2040 is projected deaths.

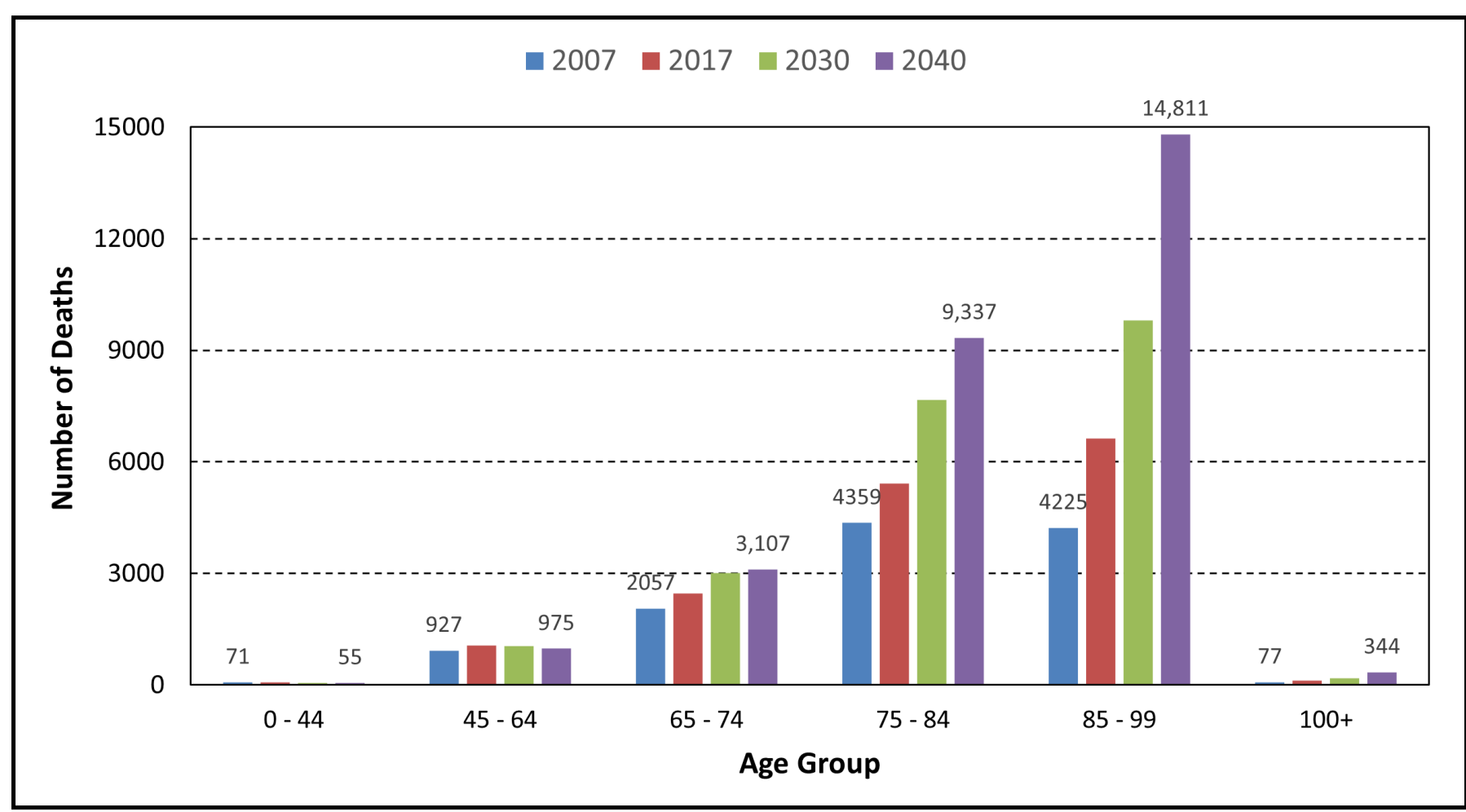

Figure 4 Projected number of people in Scotland dying from multimorbidity associated with palliative care need by age, 2017 to 2040. Note: Data for 2007 and 2017 is actual deaths; data for 2030 and 2040 is projected. Given the small number of deaths, the data for the 0 to 44 age group are too small to be visible. 
or more diseases associated with palliative care need from different disease groups.

\section{Expert consultation and consensus survey}

Thirty-four participants participated in the consultation (online supplemental file 1) and 24 completed the follow-up online survey (70.5\% response rate). Following de-duplication and synthesis of initial consultation data, 33 potential recommendations relating to nine areas were identified and included in the online survey (online supplemental file 1). Two participants were judged to have misunderstood the survey rating scale as their agreement scores were in the opposite direction of their freetext comments; these were excluded.

There was close or broad agreement that 32 of the 33 proposed recommendations across all nine areas are indicated (online supplemental file 1). Recommendations relating to digital health and person-centred care scored most highly. Agreement scores were highest for: investment in a national digital health platform $(\mathrm{M}=8.5$, $\mathrm{SD}=0.7)$; roll-out of shared electronic health and social care records across all settings $(\mathrm{M}=8.5, \mathrm{SD}=0.9)$; prioritising the patient or family perspective; $(\mathrm{M}=8.5, \mathrm{SD}=1.0)$ and systems promoting person-centred as opposed to task-oriented care $(\mathrm{M}=8.3, \mathrm{SD}=1.1)$.

\section{DISCUSSION}

\section{Principal findings}

This study projects an estimate of palliative care need in Scotland, showing that between $14 \%$ (method 2) and $20 \%$ (method 3 factoring in multimorbidities) more people may need palliative care by 2040 compared with 2017. Cancer and dementia will increase as the main underlying causes of death. For the first time, we project multimorbidity associated with palliative care needs, operationalised as the proportion of people dying from multiple chronic progressive diseases across different disease groups. We show that this proportion will rise by $60 \%$, accounting for nearly half of all palliative care deaths $(46 \%)$ by 2040 . Over half of those aged 85 and over are projected to die from multimorbidity associated with palliative care need, indicating increased complexity of needs. Experts recommended sustained investment in digital systems, such as electronic care coordination systems accessible to patients, carers and professionals, alongside prioritisation of person-centred approaches.

\section{Strengths and weaknesses}

We used a range of estimation methods accounting for both main underlying and contributory causes of deaths, which increases the robustness of our findings to inform policy and practice. The expert consultation and consensus survey ensured implications of the quantitative findings were grounded in practice. However future consultations should also consider including representatives from other professional groups including psychology, spiritual care and other specialities such as neurology and internal medicine.

Based on data available at the time of analysis, we applied linear models as we were primarily interested in projections of what may occur if recent trends continue. Our projections should not be interpreted as a forecast of what will happen, rather they provide a starting point for discussions around what may occur under different assumptions, and what actions might be needed in response. We used a disease count approach to estimate multimorbidity relevant to population palliative care need, as this is straightforward to estimate and replicate. Alternative approaches based on multimorbidity indices are increasingly common, ${ }^{17}$ though cannot be used with cause of death data alone. Our definition of multimorbidity was relatively restrictive-decedents were required to have two major illnesses from different disease groups, both of which would individually confer palliative care need. Our models draw on trends over an 11-year period, up to and including 2017, and project these trends forward. We have drawn on death registry data that relies on accurate completion of death certificates. We cannot account for changes in how cause of death is recorded over time, though previous changes have had minor impact on population level coding. ${ }^{18}$ Nor can we account for changes in medical treatments that may change the distribution of deaths by underlying cause in the future. Significant advances in the treatment of cancer or dementia might result in lower numbers dying from these diseases than is currently projected.

We also need to be prepared for more sudden increases in mortality as we have seen with COVID-19. Our estimates make no adjustment for the ongoing COVID-19 pandemic which has particularly affected people aged 75 or over who accounted for $76 \%$ of the 4649 COVIDrelated deaths registered in Scotland by 1 November $2020{ }^{19}$ Excess deaths relating to, but not directly caused by COVID-19, have also occurred. ${ }^{20}$ The long-term consequences of the virus are not yet known though recovery for some is often slow and may result in longer lasting morbidities. This spike in deaths may be followed by changes in age-specific mortality rates in following months and years. We judge that the impact of this will not change the main conclusions of this paper, that the need for palliative care will significantly rise over the next 20 years, with the number of people dying with complex needs increasing markedly.

\section{Findings in relation to existing evidence}

Our findings, that palliative care need in Scotland is projected to increase by 2040, reflect findings regarding future population palliative care need in England and Wales ${ }^{3}$ and in Ireland. ${ }^{4}$ As in these studies, in Scotland we project that the greatest increase in palliative care need will be for people aged 85 and above, reflecting an ageing population in each country. As for England and Wales, ${ }^{3}$ deaths where cancer and dementia is the main underlying cause are projected to increase, while the 
numbers dying from organ failure as the main underlying cause are projected to fall. Deaths from 'other diseases' are projected to decrease, in part due to reductions in mortality from cerebrovascular diseases and HIV, also noted in the wider literature. ${ }^{21}{ }^{22}$ In contrast to projections for England and Wales, based on underlying cause of death only, ${ }^{3}$ we did not find an increase in the proportion of people dying with palliative care needs over time (methods 2A and 2B). This may be due in part to the way deaths are recorded. In Scotland, the underlying cause has remained stable while contributory causes associated with palliative care needs have increased. Further research is needed to explore these differences.

We extended previous work, by modelling future palliative care need using both main underlying cause of death and contributory causes (method 3). This method results in a higher estimate of future palliative care need compared with other methods that examine underlying cause only. Based on method 3, we estimated that in $2017,90 \%$ of all deaths were associated with palliative care need, and that this is projected to increase to $95 \%$ by 2040 . These higher estimates are aligned with the maximal estimates reported by Murtagh and colleagues, who also used contributory cause of death to estimate palliative care in England and Wales for 2006 to 2008. ${ }^{14}$ In contrast to more conservative estimates based only on main underlying cause only, data that also accounts for contributory causes show that the vast majority of people dying now, and in the future, may benefit from a palliative care approach.

\section{Implications for clinicians, service mangers and policymakers} Multimorbidity is becoming the norm, starting earlier in the life course and rising steeply with age. ${ }^{723}$ It is widely recognised that health and social care services need to move away from a focus on care of people with single diseases towards systems that meet the needs of people with multiple diseases. ${ }^{624}$ Palliative care services also need to adapt. The hospice model is still based predominantly on care of people with a primary diagnosis of cancer; $;^{256}$ specialist services need to evolve to take account of multimorbidity, and the associated symptom burden and uncertainty, as end of life approaches. The number of people dying from dementia is is projected to be 2.8 times higher in 2040 compared with 2017, and previous research has shown that mental health comorbidities frequently occur alongside physical comorbidities. ${ }^{7}$ The palliative care service needs of patients or family members with dementia or mental health conditions need to be explicitly considered as new services are designed. More people are expected to die in care homes over the next two decades, and care homes may become the most common place of death in England and Wales by $2040 .{ }^{27}{ }^{28}$ Multimorbidity, including dementia, is common among residents. ${ }^{29-31}$ Models whereby specialist palliative care services support care homes to identify and provide palliative care for residents approaching end of life, alongside the broad expertise from multiple disciplines, need to be commonplace.
Primary care providers need to be trained as 'expert generalists ${ }^{24}$ and need to work more closely with palliative care specialists to enhance communication and coordination across the health and social care system. Electronic care coordination systems are vital to support coordinated care, enabling the sharing of key clinical information and personal preferences across all care settings and unscheduled care services; sustained investment in these systems is a priority. ${ }^{32}{ }^{33}$ As identified previously, ${ }^{34-36}$ there is a need for palliative care education and training for non-specialist health and social care workers in all settings. Training and education in the challenges of caring for people dying with multimorbidity is required. This might usefully involve exposure to primary care, geriatrics, mental health and dementia care. ${ }^{37}$ As found previously, ${ }^{28}$ greater investment in increasing and retaining the community health and social care workforce to meet the projected increases in palliative care need, is recommended.

\section{Unanswered questions and future research}

Research on dying from multiple chronic diseases requiring palliative care input is needed-which combinations of diseases are most prevalent, and how well served are those who die from multimorbidity compared with those with a single or dominant illness. Research focussing on the coexistence of mental health disorders with physical disorders at the end of life was not addressed here but needs to be prioritised in the future. We also need to better understand how well equipped health and social care professionals are to care for people with multimorbidity at the end of life; what types of care models are needed; what education, training and support is required, and for whom. Since the COVID-19 pandemic, research is required to examine the extent to which COVID-19 has influenced mortality trends and future projections of palliative care need across the UK.

\section{CONCLUSION}

By 2040, irrespective of the estimation method used, we project that many more people in Scotland will die with palliative care needs, particularly in the oldest age groups; and care complexity will increase. Current models of palliative care must adapt to meet increased need among those aged 85 and over, most dying from multiple diseases, often including dementia. There is a need for sustained investment in a national digital health and social care system, that remains person-centred, to improve care coordination and optimise palliative care for people, wherever they reside.

Author affiliations
${ }^{1}$ Research, Marie Curie Hospice Edinburgh, Edinburgh, UK
${ }^{2}$ Primary Palliative Care Research Group, Usher Institute, University of Edinburgh,
Edinburgh, UK
${ }^{3}$ Cicely Saunders Institute of Palliative Care, Policy and Rehabilitation, King's College
London, London, UK
${ }^{4}$ Public Health Scotland, Glasgow, UK

Author affiliations Edinburgh, UK

${ }^{3}$ Cicely Saunders Institute of Palliative Care, Policy and Rehabilitation, King's College

${ }^{4}$ Public Health Scotland, Glasgow, UK 
${ }^{5}$ Policy and Public Affairs, Marie Curie, Edinburgh, UK

${ }^{6}$ Health Education, Université Paris, Paris, UK

Twitter Anne M Finucane @A_Finucane and Scott A Murray @scottamurrayed

Acknowledgements We are grateful to Dr Kirsty Boyd for assistance with the expert consultation, and for commenting on a draft of this manuscript. We are very grateful to Harry Bunch (HB), Diana Robinson (DR) and Peter Buckle (PB), members of the Marie Curie Voices group, for patient and public involvement input. We thank the participants in our expert consultation for contributions at the expert consultation and for completing the follow-up survey. Special thanks to Dr Peter May at Trinity College Dublin for assisting at our consultation.

Contributors AMF, RM, AEB and SE conceived the study. AMF, AEB, SE, DC, RM, RMA, Al, SM, CE, IJH and SAM were involved in study design. DC, RMA and AMF sourced the data. DC undertook the data analysis under the supervision of AMF, $A E B, S E$ and RMA. AMF analysed data from the expert consultation with guidance from AEB and CE. AMF wrote the first draft of the manuscript, with all authors contributing to the subsequent and final drafts.

Funding This work was supported by a small grant from Marie Curie awarded on 28 February 2019 (£14 277). The grant covered funding allocated to DC at Public Health Scotland for consultation services, and to AEB and SE at Kings College London. The permanent posts of AMF and RM are funded by Marie Curie. AEB is supported by Cicely Saunders International and the Dunhill Medical Trust. CJE is funded by HEE/NIHR Senior Clinical Lectureship (ICA-SCL-2015-01-001). The views expressed are those of the author(s) and not necessarily those of the NIHR, the Department of Health and Social Care, Cicely Saunders International, the Dunhill Medical Trust or Marie Curie.

Competing interests The authors declare: financial support to DC, AEB and SE as above; and no financial relationships with any organisations that might have an interest in the submitted work in the previous 3 years. AMF and RM are employed by Marie Curie, a charity that provides palliative and end-of-life care to people with a terminal illness across the UK.

Patient consent for publication Not required.

Ethics approval The Usher Research Ethics Committee at the University of Edinburgh approved the expert consultation and consensus survey (No: 1938).

Provenance and peer review Not commissioned; externally peer reviewed.

Data availability statement All data relevant to the study are included in the article or uploaded as supplementary information. Any queries about Public Health Scotland data sources should be directed to Rosalia Munoz-Arroyo at rosalia. munoz-arroyo@phs.scot.

Supplemental material This content has been supplied by the author(s). It has not been vetted by BMJ Publishing Group Limited (BMJ) and may not have been peer-reviewed. Any opinions or recommendations discussed are solely those of the author(s) and are not endorsed by BMJ. BMJ disclaims all liability and responsibility arising from any reliance placed on the content. Where the content includes any translated material, BMJ does not warrant the accuracy and reliability of the translations (including but not limited to local regulations, clinical guidelines, terminology, drug names and drug dosages), and is not responsible for any error and/or omissions arising from translation and adaptation or otherwise.

Open access This is an open access article distributed in accordance with the Creative Commons Attribution Non Commercial (CC BY-NC 4.0) license, which permits others to distribute, remix, adapt, build upon this work non-commercially, and license their derivative works on different terms, provided the original work is properly cited, appropriate credit is given, any changes made indicated, and the use is non-commercial. See: http://creativecommons.org/licenses/by-nc/4.0/.

\section{ORCID iDs}

Anne M Finucane http://orcid.org/0000-0002-3056-059X

Scott A Murray http://orcid.org/0000-0002-6649-9428

\section{REFERENCES}

1 Foreman KJ, Marquez N, Dolgert A, et al. Forecasting life expectancy, years of life lost, and all-cause and cause-specific mortality for 250 causes of death: reference and alternative scenarios for 2016-40 for 195 countries and territories. The Lancet 2018;392:2052-90.
2 United Nations, Department of Economic and Social Affairs, Population Division. World population ageing 2015. New York; 2015. https://www.un.org/en/development/desa/population/theme/ageing/ WPA2015.asp [Accessed 21/01/2021].

3 Etkind SN, Bone AE, Gomes B. How many people will need palliative care in 2040? Past trends, future projections and implications for services. BMC Medicine 2017;15:1-10.

4 May P, Johnston BM, Normand C, et al. Population-Based palliative care planning in Ireland: how many people will live and die with serious illness to 2046? HRB Open Res 2020;2:35.

5 Academy of Medical Sciences. Multimorbidity: a priority for global health research. London; 2018. https://acmedsci.ac.uk/filedownload/39787360

6 Banerjee S. Multimorbidity--older adults need health care that can count past one. Lancet 2015;385:587-9.

7 Barnett K, Mercer SW, Norbury M, et al. Epidemiology of multimorbidity and implications for health care, research, and medical education: a cross-sectional study. Lancet 2012;380:37-43.

8 Guthrie B, Payne K, Alderson P, et al. Adapting clinical guidelines to take account of multimorbidity. BMJ 2012;345:22-4.

9 National Records of Scotland. Vital events reference tables 2017, 2018. Available: https://www.nrscotland.gov.uk/statistics-and-data/ statistics/statistics-by-theme/vital-events/deaths [Accessed 15 Aug 2018].

10 National Records of Scotland. Projected population of Scotland (2016-based), 2016. Available: https://www.nrscotland.gov.uk/ statistics-and-data/statistics/statistics-by-theme/population/ population-projections/population-projections-scotland/2016-based/ list-of-tables

11 National population projections: 2016-based projections, methodology, 2017.Office of National Statistics. Available: https:// www.ons.gov.uk/peoplepopulationandcommunity/populationandmig ration/populationprojections/compendium/nationalpopulationprojec tions/2016basedprojections [Accessed 01/07/2019].

12 Gómez-Batiste X, Martínez-Muñoz M, Blay C, et al. Identifying needs and improving palliative care of chronically ill patients: a communityoriented, population-based, public-health approach. Curr Opin Support Palliat Care 2012;6:371-8.

13 Rosenwax LK, McNamara B, Blackmore AM, et al. Estimating the size of a potential palliative care population. Palliat Med 2005; 19:556-62.

14 Murtagh FEM, Bausewein C, Verne J, et al. How many people need palliative care? A study developing and comparing methods for population-based estimates. Palliat Med 2014;28:49-58.

15 Higginson IJ, Evans CJ, Grande G, et al. Evaluating complex interventions in end of life care: the MORECare statement on good practice generated by a synthesis of transparent expert consultations and systematic reviews. BMC Med 2013;11:111.

16 Jones J, Hunter D. Consensus methods for medical and health services research. BMJ 1995;311:376.

17 Stirland LE, González-Saavedra L, Mullin DS, et al. Measuring multimorbidity beyond counting diseases: systematic review of community and population studies and guide to index choice. BMJ 2020;368:m160.

18 National Records of Scotland. The impact of the implementation of iris software for ICD-10 cause of death coding on mortality statistics in Scotland 2020. Available: https://www.nrscotland.gov.uk/files// statistics/vital-events/impact-of-implementation-iris-for-icd.pdf [Accessed 26 May 2020].

19 National records of Scotland. Deaths involving coronavirus (COVID-19) in Scotland: week 44 (26 October to 1 November), 2020. Available: https://www.nrscotland.gov.uk/covid19stats

20 Bone AE, Finucane AM, Leniz J, et al. Changing patterns of mortality during the COVID-19 pandemic: population-based modelling to understand palliative care implications. Palliat Med 2020;34:1193-201

21 Wafa HA, Wolfe CDA, Emmett E, et al. Burden of stroke in Europe. Stroke 2020;51:2418-27.

22 Public Health Scotland. HIV in Scotland: update to 31 December 2019. Publication date: 23 June 2020, 2020. Available: https://www. hps.scot.nhs.uk/web-resources-container/hiv-in-scotland-update-to31-december-2019/

23 Canizares M, Hogg-Johnson S, Gignac MAM, et al. Increasing trajectories of multimorbidity over time: birth cohort differences and the role of changes in obesity and income. The Journals of Gerontology: Series B 2018;73:1303-14.

24 WHO. Multimorbidity: technical series on safer primary care. Geneva: World health organisation 2016. Licence: CC BY-NC-SA 3.0 IGO, 2016. 
25 Allsop MJ, Ziegler LE, Mulvey MR, et al. Duration and determinants of hospice-based specialist palliative care: a national retrospective cohort study. Palliat Med 2018;32:1322-33.

26 Finucane AM, Swenson C, MacArtney JI, et al. What makes palliative care needs "complex"? A multisite sequential explanatory mixed methods study of patients referred for specialist palliative care. BMC Palliat Care 2021;20:18.

27 Bone AE, Gomes B, Etkind SN, et al. What is the impact of population ageing on the future provision of end-of-life care? population-based projections of place of death. Palliat Med 2018;32:329-36.

28 Finucane AM, Bone AE, Evans CJ, et al. The impact of population ageing on end-of-life care in Scotland: projections of place of death and recommendations for future service provision. BMC Palliat Care 2019;18:112.

29 Finucane AM, Stevenson B, Murray SA. Gradual physical decline characterises the illness trajectories of care home residents. Int $J$ Palliat Nurs 2017;23:457-61.

30 Gordon AL, Franklin M, Bradshaw L, et al. Health status of UK care home residents: a cohort study. Age Ageing 2014;43:97-103.
31 Information Services Division Scotland. Care home census for adults in Scotland: Figures for 2007-2017, 2018.

32 Finucane AM, Davydaitis D, Horseman Z, et al. Electronic care coordination systems for people with advanced progressive illness: a mixed-methods evaluation in Scottish primary care. $\mathrm{Br} J$ Gen Pract 2020;70:e20-8.

33 NHS. Coordinate my care, 2020. Available: https://www. coordinatemycare.co.uk/

34 Scottish Care. Trees that bend in the wind: exploring the experiences of front line support workers delivering palliative and end of life care 2017. Available: http://www.scottishcare.org/wp-content/uploads/ 2017/02/PEOLC-Report-final-.pdf

35 Magee C, Koffman J. Out-Of-Hours palliative care: what are the educational needs and preferences of general practitioners? BMJ Support Palliat Care 2016;6:362-8.

36 Anstey S, Powell T, Coles B, et al. Education and training to enhance end-of-life care for nursing home staff: a systematic literature review. BMJ Support Palliat Care 2016;6:353-61.

37 Oliver D. Manifesto for multimorbidity. BMJ 2018;360:k1044. 